\title{
BIOAVALIAÇÃO DO ESTADO NUTRICIONAL DO ARROZ (Oryza sativa L. VAR. IAC-165) E DO FEIJOEIRO (Phaseolus vulgaris L. VAR. CARIOCA) UTILIZANDO ${ }^{15} \mathrm{~N}$ E ${ }^{32} \mathrm{P}$
}

\author{
A.M. CalvaChe \\ Universidad Central del Ecuador - C.P. 2520, Quito - Equador \\ A.C.C. BERNARDI; F.C. OLIVEIRA \\ Departamento de Qutmica - ESALQ/USP, C.P. 9, CEP: 13418-900 - Piracicaba,SP \\ E. PRADA NETO; J.A.A. SILVA \\ Departamento de Ciencia do Solo - ESALQ/USP, C.P. 9, CEP: 13418-900 - Piracicaba,SP \\ E. MALAVOLTA \\ Centro de Energia Nuclear na Agricultura/USP, C.P. 96, CEP: 13400-970 - Piracicaba,SP
}

\begin{abstract}
RESUMO: Arroz e fejjão foram cultivados em soluções nutritivas em presença de três níveis de N, P e K. O método da bioavaliação do estado nutricional em que raizes destacadas absorvem elementos marcados, no caso presente ${ }^{15} \mathrm{~N}$ $e^{32} \mathrm{P}$, foi comparado com o de diagnose foliar. Foram tiradas duas concluszes principais: 1) os bioensaios mostraram ser um procedimento rápido e útil para o diagnóstico do estado nutricional das duas espécies, visto haver correlação significativa negativa entre absorção e produção de matéria seca; 2) a absorção de dois iśtopos pelas rárzes da planta previamente submetida a níveis diferentes de $\mathbf{N}$ e de $\mathbf{P}$ na solução foi inversamente proporcional aos teores foliares desses elementos.
\end{abstract}

Descritores: estado nutricional, bioavaliação, arroz, feijão, ${ }^{13} \mathrm{~N},{ }^{32} \mathbf{P}$.

\section{BIOEVALUATION OF THE NUTRITIONAL STATUS OF RICE (Oriza sativa L. CV. IAC-165) AND BEAN (Phaseolus vulgaris L. CV. CARIOCA) PLANTS USING ${ }^{15}$ AND ${ }^{32}$ P}

\begin{abstract}
Rice and bean plants were grown in nutrient solution in the presence of three levels of $N, P$ and $K$. The method of the hioevaluation of the nutritional status, in which excised roots are allowed to take up tagged elements, in this case ${ }^{13} \mathrm{~N}$ and ${ }^{32} \mathrm{P}$, was compared with foliar analysis. Two main conclusions were drawn: 1) the bioevaluation proved to be an useful and rapid procedure for the diagnosis of the nutritional status of both species, since there was a significant negative correlation between absorption of $N$ and $P$ and dry matter yield; 2) the uptake of the tagged ions with either element by the roots of plants grown under deficient levels of $N$ and $P$ in the nutrient solution was inversely proportional to the leaf concentration of both nutrients.
\end{abstract}

Key Words: Nutritional status, bioevaluation, rice, bean, ${ }^{13} \mathrm{~N},{ }^{32} \mathrm{P}$.

\section{INTRODUÇÃO}

Avaliar o estado nutricional de uma planta significa comparar a amostra (aspecto ou composição) com um padrão. Padrão, por sua vez, quer dizer planta ou parte dela em que todos os macro e micronutrientes aparecem em concentrações e proporções associadas com alta produtividade (MALAVOLTA et al., 1989).

Os dois métodos mais utilizados para esse tipo de comparação são a diagnose visual e a diagnose foliar. A bioavaliação do estado nutricional se baseia no princípio de que, as plantas tem uma capacidade limitada de absorver elementos do meio em que estão suas raízes. Por esse motivo, plantas bem nutridas que já absorveram um dado elemento, quando colocados na sua presença absorverão quantidades menores que os indivíduos desnutridos e com concentrações mais baixas dc elemento nos seus tecidos. Em outras palavras, dentro de limites e num período determinado, há uma relação inversa entre estado iônico interno e absorção. $O$ ensinamento tem quase 60 anos: HOAGLAND \& BROYER (1936) usavam raízes das plantas "low salt, high carbohydrate" em seus estudos de absorção iônica, visto que elas eram capazes de absorver quantidades maiores do elemento em estudo durante o período experimental. 
A bioavaliação do estado nutricional em resposta ao $\mathrm{N}$, a $\mathrm{P}$ e ao $\mathrm{K}$ faz uso dos isótopos ${ }^{15} \mathrm{~N},{ }^{32} \mathrm{P}$ e ${ }^{86} \mathrm{Rb}$, respectivamente (JONES et al., 1992; BOWEN, 1970; DIGHTON \& HARRISON, 1983; JONES et al., 1987). É que a maior sensibilidade das técnicas isotópicas permite determinar com facilidade as quantidades dos nutrientes absorvidos pelas raízes durante um periodo experimental de curta duração.

A presente contribuição se baseou em duas hipóteses:

1) a bioavaliação pode ser usada para o diagnóstico do estado nutricional do arroz e do feijoeiro;

2) no diagnóstico do estado nutricional a análise das folhas e a bioavaliação devem fornecer dados que se correlacionem.

\section{MATERIAL E MÉTODOS}

As plantas foram cultivadas em vasos de 2 litros de capacidade contendo a solução nutritiva de Johnson et al. (1957) modificada por MALAVOLTA et al. (1993). Foram usados três níveis de $\mathrm{N}(1,6 ; 4,0$ e 16 milimoles por litro), de $\mathrm{P}(0 ; 0,5$ e 2,0 milimoles) e de $\mathrm{K}(0,6 ; 3,0$ e 6,0 milimoles). Quando variava um elemento, os outros dois eram fornecidos na dose mais alta. No tratamento completo, os três nutrientes eram fornecidos nas doses mais altas. Além disso, os demais macro e micronutrientes foram integralmente fornecidos para todos tratamentos. Durante $\circ$ periodo experimental de 120 dias (transplante até colheita final), as soluções eram constantemente arejadas e foram renovadas mensalmente.

A amostragem para diagnose foliar foi feita no início do florescimento para o feijoeiro, e no início do perfilhamento para o arroz, colhendose, respectivamente, a primeira folha recém amadurecida e a folha "Y". Os teores solúveis $\left(\mathrm{N}^{-\mathrm{NO}_{3}{ }^{-} \mathrm{e}}\right.$ $\mathrm{P}-\mathrm{H}_{2} \mathrm{PO}_{4}{ }^{-}$) foram determinados de acordo com ULRICH (1948). Os teores totais, foram analisados segundo MALAVOLTA et al. (1989).

Os ensaios da bioavaliação foram feitos com a planta no fim do ciclo, quando os efeitos dos tratamentos eram mais evidentes. No bioensaio $\operatorname{com}{ }^{15} \mathrm{~N}$, as raízes destacadas, das plantas submetidas aos 3 níveis de $\mathrm{N}$, foram divididas em duas partes e, lavadas rapidamente em água corrente e depois em água destilada. Então, foram colocadas numa solução de $\mathrm{CaSO}_{4} \cdot 2 \mathrm{H}_{2} \mathrm{O} 5$ $x 10^{-4} \mathrm{M}$, durante 30 minutos (pré-tratamento). Em seguida, foram colocadas em $200 \mathrm{ml}$ de uma solução de sulfato de amônio ${ }^{15} \mathrm{~N}$ com $96 \%$ de átomos $\mathrm{em}$ excesso em sulfato de cálcio $5 \times 10^{-5} \mathrm{M}$. Depois de um período de absorção de 2 horas, com agitação por rotação de 1 minuto em intervalos de 15 minutos, as raízes foram lavadas em água corrente e secas em estufa a $70-80^{\circ} \mathrm{C}$. Determinouse a porcentagem de átomos de ${ }^{15} \mathrm{~N}$ por espectrometria de massa (FIEDLER \& PROKSCH, 1975) e calculou-se a quantidade absorvida. No ensaio para a bioavaliação do fósforo o prétratamento foi o mesmo utilizado para o N. Em seguida, as raízes foram postas a absorver durante 15 minutos $\mathrm{KH}_{2} \mathrm{PO}_{4} 5 \times 10^{-6} \mathrm{M}$ marcado com 20 $\mu \mathrm{ci}$ de ${ }^{32} \mathrm{P}$ por litro. Foram lavadas com três porções de $100 \mathrm{ml}$ de água destilada e secas em estufa a $70-80^{\circ} \mathrm{C}$. Fez-se um extrato nítricoperclórico e numa alíquota, depois de seca, fez-se a contagem do ${ }^{32} \mathrm{P}$ utilizando-se um contador Geiger-Müller, acoplado a um escalímetro.

Os resultados obtidos foram submetidos à análise de variância, e utilizou-se o teste de Tukey $(p<0,05)$ para a comparação entre médias. Foram feitas regressões entre os resultados de absorção do bioensaio e as concentrações de nutrientes na solução nutritiva. Os resultados do bioensaio foram ainda correlacionados com os teores de nutrientes totais, solúveis e acumulados na planta.

\section{RESULTADOS E DISCUSSÃO}

\section{Cultura do feijoeiro - Diagnose foliar}

Na TABELA 1 estão os teores totais e solúveis de elementos nas folhas de feijão. Observaram-se diferenças significativas entre as plantas do tratamento completo, que receberam os nutrientes nas doses mais altas, e as plantas com deficiências de N, P e K.

O teor de $N$-total nas folhas das plantas do tratamento completo é considerado como adequado de acordo com o trabalho de COBRA NETTO et al. (1971), enquanto que os teores nos tratamentos deficientes em $\mathrm{N}$ seriam considerados como deficientes. Conforme MALAVOLTA et al. (1989), os teores de $\mathrm{N}$ nos tratamentos completos e deficientes em $N$, seriam considerados como deficientes.

Os teores de P-total obedeceram à mesma tendência e segundo MALAVOLTA et al. (1989), o tratamento completo teria um teor alto. e ambos os tratamentos deficientes, teores baixos. 
TABELA 1 - Teores totais de N, P e K (\%) e solúveis de N e P $\left(\mu \mathrm{g} \cdot \mathrm{g}^{-1}\right)$ nas folhas de feijoeiro, em função dos tratamentos.

\begin{tabular}{cccccc}
\hline \hline Tratamento $\left(\mathrm{mM} . \mathrm{H}^{\mathrm{l}}\right)$ & \multicolumn{3}{c}{$\mathrm{N}$} & $\mathrm{P}$ & $\mathrm{K}$ \\
\cline { 2 - 6 } $\mathrm{N}-\mathrm{P}-\mathrm{K}$ & $\%$ & $\mu \mathrm{g} \cdot \mathrm{g}^{-1}$ & $\%$ & $\mu \mathrm{g} \cdot \mathrm{g}^{-1}$ & $\%$ \\
\hline $16,0-2,0-6,0$ & $2,47 \mathrm{a}$ & $1035 \mathrm{a}$ & $0,62 \mathrm{a}$ & $4748 \mathrm{a}$ & $2,45 \mathrm{a}$ \\
$4,0-2,0-6,0$ & $1,72 \mathrm{ab}$ & $411 \mathrm{~b}$ & - & - & - \\
$1,6-2,0-6,0$ & $1,36 \mathrm{~b}$ & $301 \mathrm{~b}$ & - & - & - \\
$16,0-0,5-6,0$ & - & - & $0,09 \mathrm{c}$ & $1006 \mathrm{c}$ & - \\
$16,0-0,0-6,0$ & - & - & $0,24 \mathrm{~b}$ & $2285 \mathrm{~b}$ & - \\
$16,0-2,0-3,0$ & - & - & - & - & $1,55 \mathrm{~b}$ \\
$16,0-2,0-0,6$ & - & - & - & - & $0,20 \mathrm{c}$ \\
\hline
\end{tabular}

Médias seguidas de letras diferentes, na mesma coluna, diferem estatisticamente a $P<0,05$.

Os teores de K-total seriam considerados como deficientes segundo COBRA NETTO et al. (1971), pois consideram adequado um valor de $4,45 \%$ de K, para a planta toda. Segundo MALAVOLTA et al. (1989), o tratamento completo teria um teor alto de $\mathrm{K}$ e os deficientes, teores baixos.

Os valores de $P$ solúvel relativamente mais altos no tratamento $0,0 \mathrm{mM} P$ que no tratamento $0,5 \mathrm{mM} P$ se devem possivelmente à menor diluição do $P$ na matéria seca acumulada, pois as plantas, antes do início dos tratamentos, receberam fósforo em baixa concentração na solução nutritiva.

Bioensaio de absorção de $\mathrm{N}$ e P: Na TABELA 2 estão os resultados da bioavaliação de $\mathrm{N}$ e $\mathrm{P}$. Foi feita uma regressão linear, entre $\circ \log (10) \mathrm{da}$ absorção de ${ }^{15} \mathrm{~N}$ da solução do bioensaio ( $\mu \mathrm{g} \mathrm{N} \cdot \mathrm{g}$ $\left.{ }^{1} \cdot h^{-1}\right)$ e a concentração de $N$ na solução nutritiva, na qual as plantas foram cultivadas. Obteve-se uma regressão linear estreita:

$\log (10) \mu \mathrm{g} \mathrm{N} \cdot \mathrm{g}^{-1} \cdot \mathrm{h}^{-1}=1,760-0,027 \mathrm{mMN}\left(\mathrm{R}^{2}=0,8\right)$

Para comprovar-se a utilidade deste método de avaliação do estado nutricional de $\mathrm{N}$ no feijoeiro, fêz-se correlações com outros métodos: teor de $\mathrm{N}$ total, teor de $\mathrm{N}$ solúvel e acumulação de $N$. Os coeficientes de correlação obtidos, entre o bioensaio e estes métodos, foram:

$\mathrm{N}$-total foliar, $\mathrm{r}=-0,95$;

$\mathrm{N}-\mathrm{NO}_{3}, \mathrm{r}=-0,88$

Acumulação de $\mathrm{N}, \mathrm{r}=-0,95$.
Nota-se que, os bioensaios de absorção radicular com ${ }^{15} \mathrm{~N}$ são uma ferramenta auxiliar para diagnosticar o estado nutricional das plantas, e estudar as necessidades de adubação. Os resultados obtidos neste ensaio concordam com os relatados por JONES et al. (1992).

A TABELA 2 mostra os resultados de absorção de ${ }^{32} \mathrm{P}$ da solução pelas raízes destacadas do feijoeiro. Os resultados originais (nM P. $\mathrm{g}^{-1} \cdot \mathrm{h}^{-1}$ ) foram transformados a $\log (10)$, segundo recomendações de HARRISON \& HELLIWELL (1979) e obteve-se uma regressão negativa com a concentração de $\mathbf{P}$ na solução nutritiva. As raízes das plantas deficientes em $P(0,0 \mathrm{mM} P)$ mostraram uma absorção do ${ }^{32} \mathrm{P}$ 6,5 vezes maior que das plantas não deficientes $(2,0 \mathrm{mM} \mathrm{P})$. A regressão obtida foi:

$\log (10) n M P \cdot g^{-1} \cdot h^{-1}=2,810-0,384 \mathrm{mMP}\left(r^{2}=0,96\right)$

As correlações obtidas, entre o ensaio de bioavaliação de $\mathrm{P}$ com outros métodos de avaliação do estado nutricional, foram as seguintes:

P-total foliar, $\mathrm{r}=-0,76$;

$\mathrm{P}_{-} \mathrm{H}_{2} \mathrm{PO}_{4}^{-}, \mathrm{r}=-0,71$;

Acumulação de $P, r=-0,93$.

Os resultados obtidos concordam com os obtidos por BOWEN (1970) e HARRISON \& HELLIWELL (1979), mostram o potencial do método dos traçadores para detectar deficiências nutricionais. 
TABELA 2 - Absorção de ${ }^{15} \mathrm{~N}$ e ${ }^{32} \mathrm{P}$ pelas raízes destacadas de feijão.

\begin{tabular}{|c|c|c|}
\hline Tratamento (mM.1-1) & Absorção de ${ }^{15} \mathrm{~N}$ & Absorção de ${ }^{32} \mathrm{P}$ \\
\hline $\mathbf{N}-\mathbf{P}-\mathbf{K}$ & $\log (10) \mu g N \cdot g^{-1} \cdot h^{-1}$ & $\log (10) n M P \cdot g^{-1} \cdot h^{-1}$ \\
\hline $16,0-2,0-6,0$ & $1,34 \mathrm{c}$ & $2,06 \mathrm{~b}$ \\
\hline $4,0-2,0-6,0$ & $1,54 \mathrm{~b}$ & - \\
\hline $1,6-2,0-6,0$ & $1,81 \mathrm{a}$ & - \\
\hline $16,0-0,5-6,0$ & - & $2,53 \mathrm{~b}$ \\
\hline $16,0-0,0-6,0$ & - & $2,87 \mathrm{a}$ \\
\hline
\end{tabular}

Médias seguidas de letras diferentes, na mesma coluna, diferem estatisticamente a $\mathrm{P}<0,05$.

TABELA 3 - Teores totais de N,P e K (\%) e solúveis de N e P $\left(\mu \mathrm{g} \cdot \mathrm{g}^{-1}\right)$ nas folhas de arroz, em função dos tratamentos.

\begin{tabular}{cccccc}
\hline \hline Tratamento $\left(\mathrm{mM} . \mathrm{I}^{-1}\right)$ & \multicolumn{3}{c}{$\mathrm{N}$} & \multicolumn{3}{c}{$\mathrm{P}$} \\
\cline { 2 - 6 } $\mathrm{N}-\mathrm{P}-\mathrm{K}$ & $\%$ & $\mu \mathrm{g} \cdot \mathrm{g}^{-1}$ & $\%$ & $\mu \mathrm{g} \cdot \mathrm{g}^{-1}$ & $\%$ \\
\hline $16,0-2,0-6,0$ & $4,28 \mathrm{a}$ & $3100 \mathrm{a}$ & $0,95 \mathrm{a}$ & 9500 & $2,04 \mathrm{a}$ \\
$4,0-2,0-6,0$ & $3,86 \mathrm{ab}$ & $3000 \mathrm{ab}$ & - & - & - \\
$1,6-2,0-6,0$ & $3,38 \mathrm{~b}$ & $1100 \mathrm{~b}$ & - & - & - \\
$16,0-0,5-6,0$ & - & - & $0,10 \mathrm{c}$ & 6500 & - \\
$16,0-0,0-6,0$ & - & - & $0,69 \mathrm{~b}$ & - & - \\
$16,0-2,0-3,0$ & - & - & - & - & $2,00 \mathrm{a}$ \\
$16,0-2,0-0,6$ & - & - & - & - & $1,85 \mathrm{~b}$ \\
\hline
\end{tabular}

Médias seguidas de letras diferentes, na mesma coluna, diferem estatisticamente a $\mathrm{P}<0,05$.

\section{Cultura do arroz - Diagnose Foliar:}

$\mathrm{Na}$ TABELA 3 estão os teores solúveis e totais de $\mathrm{N}, \mathrm{P}$ e $\mathrm{K}$ nas folhas de arroz. Os teores de $\mathrm{N}(2,5-3,0 \%)$ nas folhas estão acima e os teores de $\mathrm{K}$ estão abaixo da faixa considerada adequada por MALAVOLTA et al. (1982). Tais números parecem evidenciar a existência de um desequilibrio entre $N$ e $K$ mais acentuado nos níveis elevados desses nutrientes na solução nutritiva. O teor de $\mathbf{P}$ nas folhas está abaixo do nível adequado $(0,25-0,4 \%)$ nos tratamentos deficientes em $P\left(0,0\right.$ e $0,5 \mathrm{mM} .1^{-1}$, porém no tratamento completo $\left(2,0 \mathrm{mM} . \mathrm{l}^{-1}\right)$ está acima do adequado (MALAVOLTA et al. 1982).

A variação na produção de matéria seca total foi melhor explicada pelo teor de nitrogênio solúvel na folha $\left(\mathrm{N}-\mathrm{NO}_{3}^{-}\right)$do que pelo $\mathrm{N}$-total. A forma nítrica do nitrogênio refletiu melhor o estado nutricional do arroz, pois correlacionou-se muito bem com a produção de matéria seca, o que demonstra a possibilidade de uso destas técnicas para a avaliação do estado nutricional.

Bỉoavaliação de N e P: Na TABELA 4 estão os resultados de bioavaliação de $N$ e P. Encontrou-se uma regressão linear negativa entre o $\log (10) \mathrm{da}$ absorção de ${ }^{15} \mathrm{~N}$ da solução do bioensaio $(\mu \mathrm{g} \mathrm{N}$. $\left.\mathrm{g}^{-1} \cdot \mathrm{h}^{-1}\right)$ com a concentração do $\mathrm{N}$ na solução nutritiva (mM N.1-1), na qual as plantas foram cultivadas. A regressão obtida foi:

$\log (10) \mu \mathrm{gN} \cdot \mathrm{g}^{-1} \cdot \mathrm{h}^{-1}=1,280-0,076 \mathrm{mMN} \cdot \mathrm{I}^{-1}\left(\mathrm{R}^{2}=0,84\right)$ 
TABELA 4 - Absorção de ${ }^{15} \mathrm{~N}$ e ${ }^{32} \mathrm{P}$ pelas raízes destacadas de feijão.

\begin{tabular}{ccc}
\hline \hline Tratamento (mM.1-1) & Absorção de $15 \mathrm{~N}$ & Absorção de 32P \\
\cline { 2 - 3 } $\mathrm{N}-\mathrm{P}-\mathrm{K}$ & $\log (10) \mu \mathrm{g} \mathrm{N} \cdot \mathrm{g}^{-1} \cdot \mathrm{h}^{-1}$ & $\log (10) \mathrm{nM} \mathrm{P} \cdot \mathrm{g}^{-1} \cdot \mathrm{h}^{-1}$ \\
\hline $16,0-2,0-6,0$ & $0,11 \mathrm{c}$ & $0,50 \mathrm{~b}$ \\
$4,0-2,0-6,0$ & $0,70 \mathrm{~b}$ & - \\
$1,6-2,0-6,0$ & $1,39 \mathrm{a}$ & - \\
$16,0-0,5-6,0$ & - & $0,40 \mathrm{~b}$ \\
$16,0-0,0-6,0$ & - & $1,8 \mathrm{a}$ \\
\hline
\end{tabular}

Médias seguidas de letras diferentes, na mesma coluna, diferem estatisticamente a $\mathrm{P}<0,05$

Para verificar a utilidade do método de bioavaliação do $\mathrm{N}$, foram feitas correlações com o teor de $\mathrm{N}$-total, teor de $\mathrm{N}$-solúvel e acumulação de $N$. Os coeficientes obtidos foram :

$\mathrm{N}$-total foliar, $\mathrm{r}=-0,99$;

$\mathrm{N}-\mathrm{NO}_{3}^{-}, \mathrm{r}=-0,91$

Acumulação de $\mathrm{N}, \mathrm{r}=-0,93$.

Verifica-se, portanto, que os bioensaios de absorção radicular com ${ }^{15} \mathrm{~N}$ são úteis para diagnosticar o estado nutricional do arroz $\mathrm{e}$ avaliar as necessidades de adubação. Os resultados obtidos estão coerentes com os relacionados na literatura (JONES et al. 1992) e obedecem ao princípio do método (BINKLEY \& VITOUSE, 1992; STUART \& VAN CLEVE, 1992)

A TABELA 4 mostra os resultados de absorção de ${ }^{32} \mathrm{P}$ da solução pelas raízes destacadas do arroz. Nas raízes das plantas deficientes em $P$ $(0,0 \mathrm{mMP})$ a absorção de ${ }^{32} \mathrm{P}$ foi 12 vezes maior que nas raízes das plantas não deficientes $(0,5$ e 2,0 $\mathrm{mM} \mathrm{P.} \mathrm{l}^{-1}$ ). Os resultados originais de absorção (nM $\left.P \cdot g^{-1} \cdot h^{-1}\right)$ foram transformados a $\log (10)$ segundo recomendações de HARRISON \& HELLIWELL (1979). A regressão entre a absorção de ${ }^{32} \mathrm{P}$ e a concentração de $\mathrm{P}$ (mM P.I $\mathrm{I}^{-1}$ ) na solução nutritiva foi:

$\log (10) n M P \cdot g^{-1} \cdot h^{-1}=2,80-0,383 m M P \cdot l^{1}\left(r^{2}=0,96\right)$

CARVALHO (1987) também observou aumento nas quantidades de ${ }^{32} \mathrm{P}$ absorvidas por raízes de plantas de arroz submetidas à deficiência de P. As correlações, entre a bioavaliação de $P$ com outros métodos de avaliação do estado nutricional do $\mathrm{P}$ em arroz, forneceram os coeficientes:
P-total foliar, $r=-0,76$

$\mathrm{P}-\mathrm{H}_{2} \mathrm{PO}_{4}^{-}, \mathrm{r}=-0,91$;

Acumulação de $P, r=-0,94$.

Conclui-se, portanto, que a bioavaliação com ${ }^{15} \mathrm{~N}$ e ${ }^{32} \mathrm{P}$ refletiu muito bem o estado nutricional do arroz em $N$ e $P$, principalmente nos níveis mais deficientes desses elementos na solução, mostrando o potencial dos métodos dos traçadores isotópicos para detectar deficiências nutricionais e avaliar as necessidades de adubação.

\section{CONCLUSÕES}

1) os teores solúveis e totais de $\mathrm{N}$ e $\mathrm{P}$ na folha apresentaram alta correlação positiva com os níveis de nutriente na solução, mostrando que a diagnose foliar é um método adequado de avaliação do estado nutricional do arroz e do feijoeiro;

2) os bioensaios com ${ }^{15} \mathrm{~N}$ e ${ }^{32} \mathrm{P}$ demonstraram seu potencial como teste rápido de diagnóstico de deficiências de $N$ e $P$ nas plantas de arroz e feijoeiro, apresentando uma alta correlação significativa e negativa com a produção de matéria seca e teor de nutrientes na planta.

\section{AGRADECIMENTOS}

Participaram da elaboração e execução deste trabalho: A.A.C. Salviano, A.L.M. Menck, E.C. Brasil, F. Broetto, H.S. Pereira, J.A. Scarpare Filho, J.S. de Holanda, M.C.S. Carvalho, M.R.L. Rodrigues, R.R. Beltran, R.J.M. Hernandez, S.R. Penteado, U. Gabe, V.M. Silva, I.M.T. Piza, M.Y. Nishimura e A.A. Gallego. 


\section{REFERÊNCIAS BIBLIOGRÁFICAS}

BINKLEY, D.; VITOUSE, K.P. Soil nutrients availability. In: PEARCY, R.W.; EHLERINGER, F.; MOONEY, H.A.; RUNDEL, P.W., (Ed.), Plant physiological ecology: field methods and instrumentation. London: Chapman \& Hall, 1992. p.75-96.

BOWEN, G.D. Early detection of phosphate deficiency in plants. Communications in Soil Science and Plant Analysis, New York, v.1, n.5, p.293-298, 1970.

CARVAlHo, J.G. Efeito da deficiência de minerais na absorção de fósforo pelo arroz (Oriza sativa L.). Piracicaba, 1987. 88p. Tese (Doutorado) - Escola Superior de Agricultura "Luiz de Queiroz", Universidade de São Paulo.

COBRA NETTO, A.; ACCORSI, W.R.; MALAVOLTA, E. Estudos sobre a nutrição mineral do feijoeiro (Phaseolus vulgaris L. var. Roxinho). Anais da Escola Superior de Agricultura "Luiz de Queiroz", Piracicaba, v.28, p.257-274, 1971.

DIGHTON, J.; HARRISON, A.F. Phosphorus nutrition in lodgepole pine and Sitka spruce stande as indicated by a root bioassay. Forestry, Oxford, v.56, n.3, p.33-43, 1983 .

FIEDLER, R.; PROKSCH, G. The determination of ${ }^{15} \mathrm{~N}$ by emission and mass spectrometry in biochemical analysis. A review. Analytica Chimica Acta, Amsterdan, v.78, p.1-62, 1975.

HARRISON, A.F.; HELLIWELL, D.R. A bioassay for comparing phosphorus availability in soils. Journal of Applied Ecology, Oxford, v.16, p.491-505, 1979.

HOAGLAND, D.R.; BROYER, T.C. General nature of the process of salt acumulation by roots with description of experimental methods. Plant Physiology, Rockville, v.11, p.471-507, 1936.

JONES, H.E.; HARRISON, A.F.; DIGHTON, J. A ${ }^{86} \mathrm{Rb}$ bioassay to determine the potassium status of trees. New Phytologist, Cambrigde, v.107, n.4, p.695-708, 1987.
JONES, H.E.; QUARMBY, C.; HARRISON, A.F. Evaluation of the sensitivity of a ${ }^{19} \mathrm{~N}$ test for nitrogen deficiency in plants. Communications in Soil Science and Plant Analysis, New YorK, v.23, p.1333-1343, 1992.

MALAVOLTA, E.; BOARETTO, A.E.; MURAOKA, T. Avaliação do estado nutricional do arroz e do feijoeiro. Piracicaba: CENA, 1993.28p.

MALAVOLTA, E.; VITTI, G.C.; OLIVEIRA, S.A. Avaliação do estado nutricional das plantas: princípios e aplicações. Piracicaba: Associação Brasileira para Pesquisa da Potassa e do Fosfato, 1989. 201 p.

MALAVOLTA, E.; PAULETTO, E.A.; FRETTAS, J.R.; CAVALCANTI, L.F.; LIVA, M.L.; FIORE, M.F.; PRIMAVESI, O.; FONSECA, S.M.; CABRAL, C.P. Estudos sobre a nutrição mineral do arroz. XVII. Exigências nutricionais das variedades IAC-164 e IAC-165". Anais da Escola Superior de Agricultura "Luiz de Queiroz", Piracicaba, v.39, n.1, p.411-424, 1982.

STUART, F.; VAN CLEVE, K. Approaches to studying nutrient uptake, use and loss in plants. In: PEARCY, R.W.; EHLERINGER, F.; MOONEY, H.A.; RUNDEL, P.W., (Ed.). Plant physiological ecology: field methods and instrumentation. London: Chapman \& Hall, 1992. p.189-207.

ULRICH, A. Plant Analysis-methods and interpretation of results. In: KITCHEN, H.B., (Ed.). Diagnostic techniques for soils and plants. Washington: The American Potash Institute, 1948. p.157-198.

Recebido para publicação em 02.03.94

Aceito para publicação em 20.06.94 